\title{
Lengua y cultura ante la metamorfosis de la educación de estos tiempos
}

\author{
» Language and culture before the metamorphosis \\ of education in these times
}

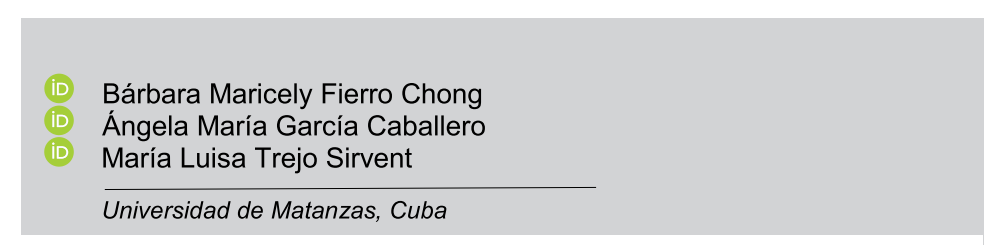

\section{Resumen}

El proceso investigativo de enseñar y aprender lenguas en la formación de docentes en las universidades es mostrado en este artículo desde la perspectiva de un enfoque integrador de lengua y cultura, a partir de las bases del enfoque cognitivo, comunicativo y sociocultural de la enseñanza de la lengua y la literatura, con la inclusión de la innovación como proceso que acompaña a las ciencias sociales y humanísticas. Se emplearon métodos de carácter cualitativo que posibilitó revelar el carácter transformador y de proceso en el aprendizaje creativo en estudiantes de la carrera profesoral de Lengua y Literatura en la Universidad de Matanzas. Las autoras realizaron un estudio de campo que propició articular alianzas universidad-sociedad, desde la investigación.

Palabras clave: innovación, educación, identidad, lengua, literatura.

\begin{abstract}
The investigative process of to teach and to learn languages in the formation of educational in the universities is shown in this article from the perspective of an integrative focus of language and culture, starting from the bases of the focus cognitivo, talkative and sociocultural of the teaching of the language and the literature, with the inclusion of the innovation like process that it accompanies to the social and humanistic sciences, methods of qualitative character were used that facilitated to reveal the character transformer and of process in the creative learning in students of the professorial career of Language and Literature in the University of Matanzas. The authors carried out a field study that propitiated to articulate alliances university - society, from the investigation
\end{abstract}

Keywords: innovation, education, identity, language, literature.

\section{Cómo citar este artículo:}

Fierro Chong, B., García Caballero, A. y Trejo Sirvent, M. (2020). Lengua y cultura ante la metamorfosis de la educación de estos tiempos. Amauta, 18 (35), pp.39-5. DOI: https://doi.org/10.15648/am.35.2020.4

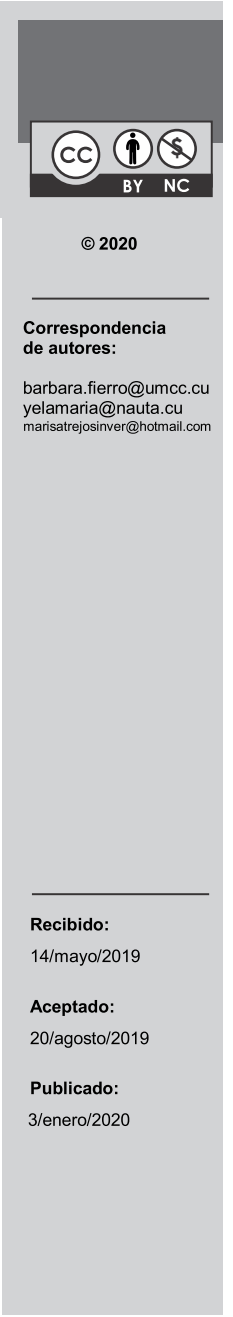




\section{Introducción}

La humanidad en las puertas de la tercera década del siglo XXI, (Morín \& Delgado 2017) está viviendo un cambio de época, marcado por acontecimientos globales que indican a la humanidad caminos éticos que posibiliten dar respuesta a las grandes contradicciones que caracterizan el momento actual; la ciencia y la tecnología han de encontrar una respuesta que frene las consecuencias nefastas de los trastornos provocados por el cambio climático y las pandemias. Se ha puesto a prueba la capacidad de resiliencia, de comunicación y de pensar juntos, soluciones globales.

Las universidades lideran la ciencia, la tecnología y la innovación para mitigar las consecuencias que en el desarrollo humano generan las grandes conversiones sociales de este siglo, se exige de un enfoque inter y multidisciplinar, de ahí que es necesario el trabajo en redes y las alianzas entre cuerpos académicos de investigación para solucionar problemas que atañen a diferentes comunidades, más allá de sus ideologías, intereses, escenarios de convivencia, tales como: la cultura de paz, el enfrentamiento a las grandes pandemias que ponen en riesgo la vida humana, la oposición a las guerras de colonización y el genocidio de los grandes imperios frente a los pueblos que luchan por su soberanía, todos ellos mediatizados por la comunicación, y en ella la transformación que se opera en la comprensión y producción de significados por la presencia y efecto de las Nuevas Tecnologías de la Información y las Comunicaciones.

Para la comprensión del mundo, de las ideas y el triunfo del bien, la lectura es acompañamiento imprescindible porque condiciona el pensamiento de los individuos para las transformaciones, con ello se reduce la resistencia ante los cambios. Se consolida la construcción de significados compartidos y el consenso, el aprovechamiento de oportunidades como un recurso clave para el desarrollo de la competencia comunicativa en los diferentes niveles de educación; la exorbitante presencia de los bulos y face news ponen a los lectores de miles de redes al borde de la credulidad de los datos.

Es más necesario que nunca seguir repensando la lengua que se enseña y se aprende a la luz de los nuevos tiempos en un complejo entramado sociocultural, ante la avalancha de los medios informales; la adquisición de nuevos saberes por 
los docentes implica acercarse al estudio de los procesos culturales y de comunicación desde la perspectiva de la ciencia.

La mirada crítica a los referentes epistemológicos que movilizan el pensar, sentir, vivir la lengua (García, Fierro \& Montaño, 2019) en la formación de docentes de lengua materna en Cuba posibilitaron identificar los referentes de la alfabetización académica, (Carlino, 2008), los enfoques comunicativos (Cassany \& Morales, 2008) y el enfoque cognitivo, comunicativo y sociocultural de la enseñanza de la lengua y la literatura (Roméu, 2007), así como el interaccionismo discursivo (Bronckart, 2012), la creatividad en la enseñanza de las lenguas (Trejo, 2019), y el valor de las investigaciones sobre los procesos de lectura y escritura en la educación superior (Navarro et. al., 2016).

En el proceso de investigación realizado por las autoras de este artículo en sus referidos campos de actuación universitaria y de cara a la lengua que se enseña en sus países, a saber: México (Trejo, 2015, 2019) y Cuba (Fierro y García, 2017, 2019), se identificaron y asumieron los referentes hallados en Pardo (1987), Roméu (2007), Montaño y Abello (2015), MRC (2001), entre otros.

Diversas definiciones fueron encontradas sobre competencia comunicativa, al respecto se destaca la ofrecida por Pardo (1987)

"Entendemos por competencia comunicativa el conjunto y estructura de capacidades de orden mental, motor y 'sensorial' indispensables a los participantes en el proceso comunicativo para la comprensión y producción de enunciado contextualmente apropiados", (1987, p. 325).

El Marco Europeo Común de Referencia señala que la competencia comunicativa comprende varios componentes: el lingüístico, el sociolingüístico y el pragmático. Estos corresponden a destrezas y habilidades. En el Marco Europeo Común de Referencia para las Lenguas (CVD, 2001) el Centro Virtual Cervantes, 2001, online) señala que la competencia lingüística es un componente que incluye:

los conocimientos y las destrezas léxicas, fonológicas y sintácticas, y otras dimensiones de la lengua como sistema, independientemente del valor sociolingüístico de sus variantes y de las funciones pragmáticas de sus realizaciones... También se puede considerar que la organización cognitiva del vocabulario y el almacenamiento de expresiones, etc. dependen, entre otras cosas, de las características culturales de la comunidad o 
comunidades donde se ha socializado el individuo y donde se ha producido su aprendizaje. (Centro Virtual Cervantes, 2001, online).

También indica que las competencias pragmáticas:

tienen que ver con el uso funcional de los recursos lingüísticos (producción de funciones de lengua, de actos de habla) sobre la base de guiones 0 escenarios de intercambios comunicativos. También tienen que ver con el dominio del discurso, la cohesión y la coherencia, la identificación de tipos y formas de texto, la ironía y la parodia. Respecto a este componente, incluso más que en el caso del componente lingüístico, apenas es necesario resaltar el gran impacto que ejercen las interacciones y los entornos culturales en el que se desarrollan las mencionadas capacidades (Centro Virtual Cervantes, 2001, online).

Los aportes de Bronckart (2012), sobre el interaccionismo sociodiscursivo constituyen clave y punto de partida de las posiciones actuales de la enseñanza de la lengua en Cuba, quien enfatiza en la enseñanza de las lenguas para una construcción de las capacidades textuales centrado en la relación entre las actividades humanas y los discursos.

De manera que, los contenidos, enfoques y vías para la enseñanza de lenguas en la educación superior en la formación de los docentes, se organiza atendiendo a la relación discurso, cognición y sociedad, revelando el papel de lo sociodiscursivo y lo sociocultural, pero también centrado en la naturaleza interdisciplinaria, el valor de la lectura, de los procesos de comprensión y construcción de significados en diversas tipologías textuales, con un valor particular de la literatura.

Un estudio de investigadores liderado por Navarro, F. (2016) mostró la tendencia de los estudios de lectura y escritura en América Latina, al examinar la producción científica publicada en revistas de Colombia, Argentina, Chile, Brasil, y que revelan que este es un campo disciplinar de notable interés para las ciencias sociales y humanas, en el que era posible identificar "denominaciones diversas (como 'alfabetización académica', 'análisis del discurso académico' o 'didáctica de la escritura en educación superior') ... con especial incidencia de los estudios del discurso, la lingüística aplicada, la psicología, las ciencias de la educación y las didácticas específicas" (2016, pp. 101). 
Este estudio identifica como complejo y diverso el panorama en las universidades de América Latina, en que se toma lo mejor de la tradición cultural y las tendencias del mundo actual, el efecto que producen las tecnologías en los conocimientos, las necesidades de estrategias cognitivas y metacognitivas para la adquisición de los saberes y la toma de posición, orientación valorativa y crítica, estratégica en la preparación del potencial humano ante los retos de la ciencia y la técnica (Núñez, 2017).

Ante las exigencias de la educación de “... estimular la inteligencia y las capacidades potenciales de niños y jóvenes, y cultivar el territorio aún más impalpable de los sentimientos" (Pogolotti, 2019); la lengua y con ella la lectura, como umbral del conocimiento y disfrute, es un contenido de naturaleza particular, para la educación de la sensibilidad y la emocionalidad, esencias de la ética y el humanismo.

La literatura en su naturaleza dialógica, de alta influencia socioafectiva ocupa un lugar no desdeñable para llegar a ese "territorio aún más impalpable" al que se refiere la ensayista Pogolotti, porque facilita que los aprendizajes sean renovables. Por eso, en términos de apropiación y generación de saberes del área de las humanidades y las ciencias sociales, el aprendizaje creativo, la innovación y las cualidades creativas poseen relevancia en las investigaciones desarrolladas por el Cuerpo Académico "Paradigmas Educativos y la Enseñanza de Lenguas" (Consolidado), liderado por la Dra. Trejo Sirvent, en la Facultad de Lenguas Campus Tuxtla, Chiapas México y el proyecto de Investigación "La enseñanza aprendizaje de las lenguas y la literatura en la Educación de la personalidad" desarrollado por un grupo de investigadores de la Universidad de Matanzas, Cuba. Un elemento común entre ambas investigaciones radica en la atención a la actividad comunicativa, en tanto reconocen que enseñar lengua es enseñar cultura, constructo de identidad que, implica en primera instancia la identidad lingüística, ambas pueden verse sacudidas y desplazadas del imaginario ante los cambios que se producen en términos de comunicación, lo que requiere, repensar los roles y en ellas la preparación de los recursos humanos.

La idea de Moreno acerca del aprendizaje es oportuna en este contexto:

El aprendizaje es también un camino. Podemos abreviarlo en las páginas de un libro, transformarlo en una aventura o hacer de él un viaje organizado. 
Los resultados que obtengamos serán muy diferentes según el método elegido, como también lo será el nivel de placer o de aburrimiento que a través de él experimentemos. (Moreno, 1993, pp. 26).

Es responsabilidad social de las universidades en la formación de sus profesionales, contribuir, desde la ciencia y la innovación (Saborido, 2018), a la introducción de vías para la invención, la creación en el aprendizaje ante los cambios que se viven a nivel global y su efecto a nivel local, en este proceso la investigación sobre enseñar lengua, y los procesos de lectura y escritura, deben atender enfoques de la lengua y la literatura que reporten innovaciones.

Al respecto, es pertinente el desarrollo del aprendizaje, particularmente el aprendizaje creativo. Si bien es un camino, con bifurcaciones donde convergen experiencias, tradiciones, influencias, la labor orientadora de la escuela y la figura del maestro han sido fundamentales, pero van más allá. Es importante destacar que: "La creatividad no se produce dentro de la cabeza de las personas, sino en la interacción entre los pensamientos de una persona y un contexto sociocultural. Es un fenómeno sistémico, más que individual" (Csikszentmihalyi, 1998, pp. 41). Tal como apunta Gardner

El aprendizaje no tiene por qué darse únicamente entre las cuatro paredes de la escuela. La tecnología nos puede llevar por todo el mundo sin tener que salir de casa. Los ciudadanos y las instituciones de una comunidad pueden hacer contribuciones importantes a la educación de sus niños y jóvenes. Estas contribuciones pueden empezar con viajes de estudio, pero no tienen -no deberían- por qué acabar aquí. (Gardner, (2000, p. 157).

En ocasiones, prima el criterio de que las clases de lenguas reiteran contenidos, sin embargo, en consecuencia, con lo anterior, la formación de maestros de esta área enfrenta el desafío de construir y renovar las formas de enseñar el mundo y sus relaciones desde lectura y la escritura.

Los docentes tienen el reto de desarrollar lo que Albistegui, (2009) llama la tercera capacidad: es la que nos habilita como innovadores: "es la de hacer posibles lo nuevo por primera vez. Esta capacidad está asociada directamente con la habilidad para llevar adelante algo que no existe y hacerlo realidad, aunque sea con múltiples defectos en su primera versión" (Albistegui, $(2009$, p. 10). 
El uso de las TIC (Tecnologías de la Educación y las Comunicaciones) es una de las aristas para la aplicación de la innovación (Aula Planeta, 205), en la enseñanza de la lengua materna, que implicó un trabajo de mesa, de coordinación y búsqueda de información por parte de estudiantes y profesores en el equipo de investigación, sobre la base de las ideas de Hernández, \& Alvarado, (2012), Alarcón, (2016), Núñez (2017).

Por otra parte, se considera con la Unesco que:

la innovación se ha vinculado, por una parte, con la incorporación de las nuevas Tecnologías de la Información y la Comunicación (TIC) en la Educación y, por otra, con el protagonismo de las instituciones educativas y docentes en el cambio 'desde abajo'. En uno u otro caso, se generan dinámicas de intercambio y trabajo compartido, como el caso de comunidades educativas o redes pedagógicas (Unesco, 2016, pp. 12).

Si bien es cierto que en el espacio formativo del aula se aprovechan las ventajas de las nuevas tecnologías, también la aplicación de métodos de enseñanzaaprendizaje, sustentados en el enfoque integrador, facilita ante todo un cambio de mentalidad para lograr un aprendizaje significativo, participativo, creativo y abierto a la recepción de los escolares y estudiantes.

\section{Metodología y métodos}

Se asumió en la investigación un enfoque cualitativo que posibilitó articular el método de sistematización de experiencias aportadas por las autoras desde los colectivos de investigación dedicados a los estudios disciplinares e interdisciplinares de lengua y lectura en la educación universitaria, y que poseen puntos de contacto y comunidad de intereses. La aplicación de métodos teóricos y empíricos como: el análisis y la síntesis, la modelación, la observación, entrevistas a profundidad, revisión de documentos, análisis de los productos de la actividad, favoreció evaluar los resultados de las tareas de investigación.

La experiencia de trabajos en el campo de la didáctica de las lenguas y la literatura de las tres investigadoras desde 2017 y consolidados entre 2018 y 2019 por el trabajo en redes de conocimientos conformó la base conceptual, teórica y didáctica del estudio. 
Los aportes de Trejo (2015) y de Torres, (2016), acerca de los métodos de investigación también fueron sustento en este resultado, incorporados a la reflexión de la investigación realizada en la Universidad de Matanzas enriquecieron los referentes para la observación y análisis de la información sobre la transformación operada en las unidades de análisis tomadas.

Al respecto, fue de pertinencia para este estudio el trabajo colaborativo de las tres investigadoras que suscriben este resultado, desde su contexto sociocultural de ampliación y complementariedad a los referentes teóricos a partir del rescate en su investigación de campo en la didáctica de las lenguas.

El procedimiento seguido se sustentó en que el currículo de la formación inicial y permanente de los docentes debe caracterizarse por su flexibilidad para incorporar nuevas ideas, la que condujo a incorporar en el currículo optativo/electivo y propio de la carrera profesoral de Español y Literatura en la Universidad de Matanzas en la etapa 2016-2019 programas formativos articulados con la actividad de la práctica laboral investigativa y la extensión universitaria que fomentaran en los estudiantes de pregrado una visión renovadora de los "aprendizajes" de la literatura a favor de la inclusión educativa, la atención a la diversidad, el empleo de las tecnologías y las posibilidades innovadoras de la enseñanza-aprendizaje de la lengua derivada de los aprendizajes renovados de la lectura literaria.

Se elaboraron como indicadores para evaluar y controlar el aprendizaje de los contenidos: independencia cognoscitiva, originalidad del resultado, uso de las tecnologías, trabajo cooperado, socialización y relación con el ejercicio de la profesión, en el espacio de la actividad académica, laboral investigativa y extensionista.

La recogida de datos se realizó mediante la observación, revisión de los productos de la actividad, trabajo cooperado, la activación de las experiencias y el encadenamiento reflexivo de los estudiantes con sus colegas de los departamentos docentes de sus unidades de trabajo.

Fueron necesarios en la metodología seguida los grupos de discusión como espacios de construcción de nuevos conocimientos, profundización en la categorización asumida en la enseñanza de la lengua con carácter sociodiscursivo, la permanencia en el trabajo de campo con los estudiantes, lo que 
posibilitó eludir el "efecto de halo" en los resultados obtenidos y la verificación de las conclusiones.

\section{Resultados y discusión}

En la formación de profesores de Español-Literatura en Cuba, se parte del fin social de la escuela para la que se prepara, e implica desarrollar aprendizajes creativos desde estos contenidos. En este sentido, se tuvo en cuenta la idea de Mariño, J. acerca de la creatividad como un objetivo de la enseñanza el desarrollo de la creatividad de los estudiantes de los diferentes niveles de enseñanza. Mariño (2002, pp. 6).

Derivado de los resultados de investigación del Proyecto La enseñanza aprendizaje de las lenguas y la literatura en la educación de la personalidad, (2017- 2020) en la Universidad de Matanzas, en la formación de pregrado de la carrera Español -Literatura, se encaminaron acciones investigativas que posibilitaron: desarrollar en los estudiantes de los años terminales una mirada innovadora de la lectura y la escritura ante las obras literarias ya estudiadas, una reinvención de los caminos de leer y disfrutar tomando como escenario su práctica laboral investigativa y la actividad extensionista.

En el curso 2016-2017, los estudiantes de la carrera Español-Literatura en su año terminal desarrollaron un ejercicio profesional integrador en la asignatura Análisis del discurso:

- Ayudas didácticas para enseñar y aprender", consistente en un módulo de materiales con apoyos de presentaciones digitales, elaboración de mapas conceptuales y esquemas para la comprensión de los contenidos del análisis del discurso.

Esta actividad se ejecutó de forma planificada durante un curso escolar, en un trabajo cooperado y colaborativo. Se organizó el trabajo en dúos lo que favorecía la colaboración, la confrontación de ideas, la toma de decisiones y la creatividad en la construcción de los nuevos saberes con un enfoque profesional pedagógico.

Se midieron los indicadores para evaluar y controlar el aprendizaje de los contenidos, ya mencionados, en tres cortes durante el curso en una escala 
ascendente del 1 al 5, mediante la observación, registro de las actividades, intercambio de ideas, las interrogantes de las estudiantes y sus comentarios, derivados también del encadenamiento metodológico con sus respectivos escenarios de la práctica laboral e investigativa.

Los estudiantes participantes mostraron paulatinamente un enriquecimiento de sus conocimientos, vivencias, así como de sus valores y de su creatividad, en el orden cultural, metodológico y educativo. Las ayudas didácticas pasaron a formar parte del material de apoyo audiovisual a la enseñanza de la asignatura Español-Literatura en las escuelas donde las estudiantes realizaban su práctica laboral.

En cuanto a la independencia cognoscitiva se mostraron sus puntos de vista, apreciaciones resultado de los conocimientos y habilidades adquiridas durante la carrera, la originalidad del resultado se mostró en la forma en que lo organizaron para cumplir los principios metodológicos de asequibilidad y propiciar el acceso a la información de los usuarios, el uso de las tecnologías reveló la incorporación de los conocimientos y habilidades con diferentes aplicaciones, el trabajo cooperado implicó una dosis de trabajo colaborativo en cada equipo, el aprovechamiento de las redes de conocimientos, el correo y el chat para el intercambio y la toma de decisiones, mientras que la socialización se mostró ascendente en los semestres segundo y tercero, dado que fue el momento en que experimentaron mayores posibilidades de presentación en las Jornadas científicas estudiantiles, sesiones de trabajo metodológico en los departamentos docentes y en el Fórum de Ciencia y Técnica de la Facultad de Idiomas y a nivel de la universidad, la relación con el ejercicio de la profesión se manifestó por encima de los dos puntos para ubicarse en un nivel máximo por cuanto este resultado fue de utilidad para sus unidades de práctica laboral. 
Gráfico 1. Comportamiento de la transformación de las estudiantes en las “ayudas didácticas”. 2019.

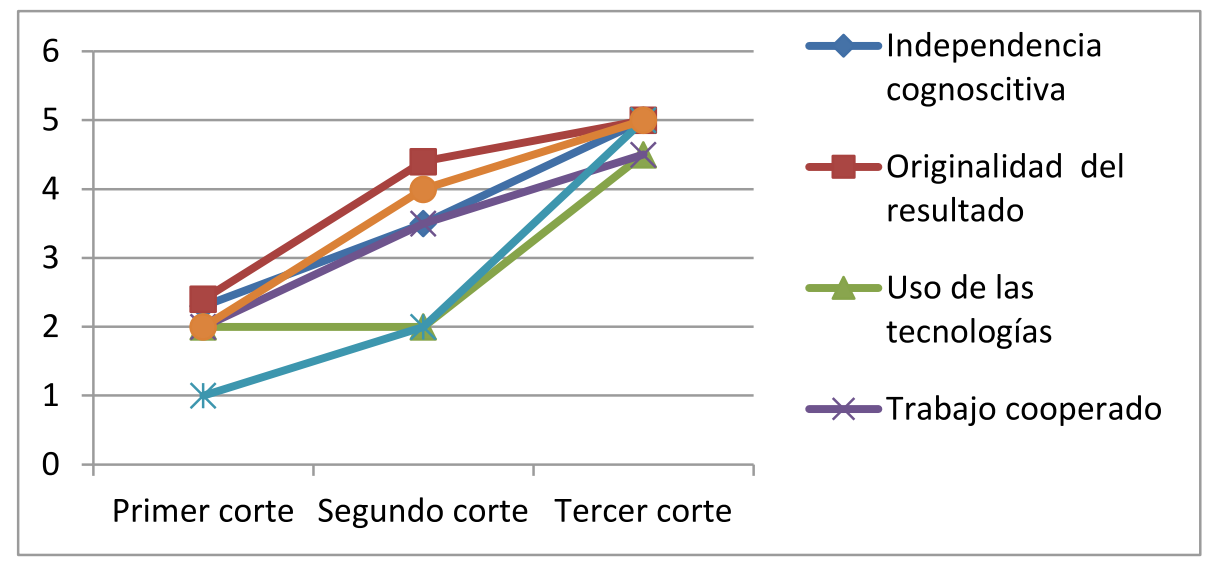

Fuente: elaboración propia.

En el curso 2017- 2018, los estudiantes de la carrera Español-Literatura, en su año terminal, desarrollaron un ejercicio profesional integrador en la asignatura Análisis del discurso consistente en la integración de saberes desde el abordaje "diferente" de la obra literaria El Ingenioso Hidalgo Don Quijote de la Mancha de Miguel de Cervantes y Saavedra.

La realización de ejercicios profesionales integradores por dos estudiantes, posibilitó seguir un estudio de caso. Este resultado está conformado por los productos informáticos Página web y video como formas de evaluación de tareas de aprendizaje: Molinos o gigantes: ¿qué ves tú?”. Una mirada al texto cervantino para la escuela", atendiendo a los indicadores Independencia cognoscitiva, Originalidad del resultado, Uso de las tecnologías, Trabajo cooperado, Socialización, Relación con el ejercicio de la profesión, para evaluar y controlar el aprendizaje de los contenidos.

En una escala del 1 al 5, en que este es el valor más alto, el comportamiento está por encima de 4, las ligeras variaciones están dadas por el uso de las tecnologías, lo que implicó que el trabajo cooperado también incluyera un aprendizaje colaborativo en que participaron otros agentes. En este caso se 
destaca la socialización y la relación con el ejercicio de la profesión, dado por los siguientes aspectos:

La socialización se expresó en: Premio en la Jornada Científica Estudiantil de la Facultad de Idiomas y la Universidad de Matanzas, obtención de tres premios en el Fórum Nacional de Estudiantes de Ciencias Pedagógicas, 2018, por la originalidad, el tratamiento de la literatura, y la innovación, presentación en el Congreso Internacional Pedagogía 2019, La Habana, Cuba y la publicación en las memorias del evento.

En cuanto a la relación con el ejercicio de la profesión se destaca su empleo como medios de enseñanza de las clases de Español-Literatura en preuniversitario en dos municipios de la provincia de Matanzas.

Grafico 2. El Comportamiento de los estudiantes en la transformación en el uso de las tecnologías para la enseñanza de El Quijote, de Miguel de Cervantes. 2019.

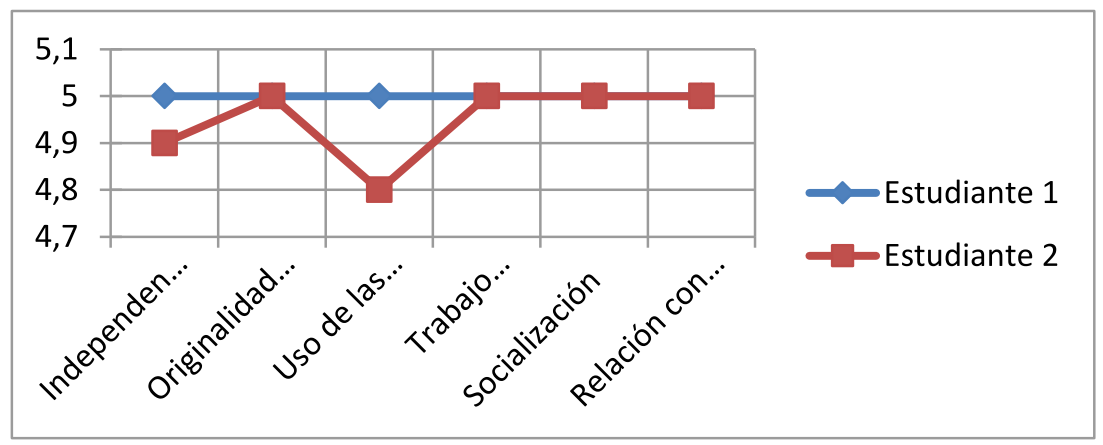

Fuente: elaboración propia.

En el proceso de valoración de los resultados de investigación, realizada en la tarea de Leer y escribir desde la universidad, se destacan como logros:

- Una competencia comunicativa que transita de la descripción y explicación de los hechos lingüísticos hacia la apreciación, reflexión y valoración textual y contextual, de carácter sociodiscursivo. 
- Prácticas discursivas en las universidades que trascienden lo académico para articular lo investigativo y extensionista en la actividad comunicativa profesional.

- Reflexión metacognitiva de los estudiantes en sus centros de práctica laboral de secundaria básica y preuniversitario, posibilitó la socialización de otras miradas a los textos en diversas tipologías textuales con empleo de las tecnologías y el desarrollo de un pensamiento crítico y creativo.

Las acciones emprendidas en la investigación con la intencionalidad didáctica en la formación de docentes, dada la flexibilidad que brinda el plan de estudio evidencia que hay vías en la actividad de innovación para lograr la metamorfosis de la clase de lenguas cuando se fomenta la inteligencia y las capacidades creadoras, y se aprovechan las diversas lecturas de la literatura para, al decir de Pogolotti "cultivar el territorio aún más impalpable de los sentimientos" (2019), como camino para la resiliencia.

\section{Conclusiones}

La innovación educativa en la enseñanza de las lenguas es consustancial al desarrollo de la creatividad que se revela en el proceso de conformación de la actividad comunicativa, de manera que la acción renovadora desde los escenarios formativos de la educación superior posee pertinencia para dar respuesta a los permanentes desafíos de la sociedad contemporánea en los aprendizajes de lenguas. Toda actividad innovadora implica la activación de las cualidades personológicas del docente en los contextos universitarios como cuna de la cultura y el pensamiento.

\section{Referencias bibliográficas}

Alarcón, R., (2016). Universidad innovadora por un desarrollo humano sostenible: mirando al 2030, Conferencia en Congreso Universidad 2016, la Habana, en <http://www.congresouniversidad.cu> [26/06/2017]. 
Albistegui, M. (2009). Creatividad e Innovación: Nuevas ideas, viejos principios Recuperado de https://www.google.com.mx/

Aula Planeta (2015). El aula creativa, cada vez más cerca de las escuelas españolas. Recuperado de http://www.aulaplaneta.com/2015/01/07/noticias-sobre-educacion/el-aulacreativa-cada-vez-mas-cerca-de-las-escuelas-espanolas-infografia/.

Bronckart, J. (2012). En las fronteras del Interaccionismo socio-discursivo: aspectos lingüísticos, didácticos y psicológicos. In: D. Riestra, S.M. Tapia \& M.V. Goicoechea. Terceras Jornadas Internacionales de Investigación y Prácticas en Didáctica de las lenguas y las literaturas: BarilochePatagonia, Argentina.

Carlino, P. (2008). Leer y escribir en la universidad, una nueva cultura ¿por qué es necesaria la alfabetización académica. Los desafíos de la lectura y la escritura en la educación superior: caminos posibles, 159194.http://www.aacademica.org/instituto.de.lingoistica.de.la.universidad .de.buenos.aires/box/paula.carlino/74.pdf. Recuperado el 18 de mayo de 2017

Casañas, M. (2015). La investigación en la educación superior en América Latina. Págs. 67-78. En: revista Amauta. Vol. 13, no. 25. Enero-junio. Universidad del Atlántico, Barranquilla, Colombia.

Cassany, D. \& Morales O. (2008). Leer y escribir en la universidad: Hacia la lectura y la escritura crítica de géneros científicos, en: Revista Memoralia, $\quad$ http://www.saber.ula.ve/handle/123456789/16457. Recuperado el 18 de mayo de 2017.

Cet (2011). Comunicación, lenguaje, lengua y habla. Recuperado de http://www.bebesymas.com/desarrollo/comunicacion-lenguaje-lengua-yhabla.

Csikszentmihalyi, M. (1998). Creatividad. El fluir y la psicología del descubrimiento y la invención. Barcelona: Paidós.

Fierro, B., García A. \& Díaz, L. (2018). El proceso de perfeccionamiento de la enseñanza de la lengua materna y la literatura: retos a la investigación, desarrollo e innovación. Curso en el XII Simposio de Pensamiento, 
Educación y Cultura. Universidad de Matanzas. Disponible en http://citc.umcc.cu

García, A., Fierro, B. \& Montaño, J. (2019). Pensar, sentir y vivir la lengua en la actividad comunicativa pedagógica. Pp. 1 -11. Revista científicoMetodológica Varona, No. 69, septiembre - diciembre, 2019. ISSN: 19928238

García, A. \& Fierro, B. (2017). La competencia analítico-textual discursiva: un aprendizaje permanente. Revista Atenas, Vol. 1 Nro. 37. Disponible en http://atenas.mes.cu

Gardner, H. (2000). La educación de la mente y el conocimiento de las disciplinas. Barcelona: Ediciones Paidós Ibérica.

Hernández, A. \& Alvarado, G. (2012). Transformación de las prácticas pedagógicas a través de nuevos dispositivos de comunicación y la escritura de textos argumentativos. Revista Iberoamericana de Educación. No. 59, marzo-agosto. Universidad Central, Colombia.

Mariño, C. (2002). Aprendizaje Creativo: Una alternativa en la formación del profesional en la educación. Recuperado de

http://es.calameo.com/read/000233168f408faec56e2.

Montaño, J. \& Abello, A. (2015). Leer y escribir. ¡Tarea de todos! La Habana: Pueblo y Educación.

Moreno, M. (1993). Los Temas transversales: una enseñanza mirando hacia delante, pp. 10-43. Busquets, M., Cainzos, M., Fernández, T., Leal A., Moreno, M. y Sastre, G. (1993). Los temas transversales. Madrid: Santillana.

Morín, E. \& Delgado, C. (2017). Reinventar la educación. Abrir los caminos hacia la metamorfosis. La Habana: Universidad de la Habana.

Navarro, F. (2016). Panorama histórico y contrastivo de los estudios sobre lectura y escritura en educación superior publicados en América Latina. Pág. 100-126. Revista Signos. Estudios de lingüística. PUCV, Chile, DOI: 10.4067/S0718-09342016000400006,49 (S1)

Núñez, J. (2017). La didáctica de la educación superior en el siglo XXI y el desarrollo sostenible, conocimiento, universidad y desafíos del desarrollo. En: "La didáctica de la Educación Superior ante los retos del siglo XXI", 
compiladora Verena Páez, sello Editor Educación Cubana, publicado en www.ucpejv.edu.cu

Pardo, J. (1987). Centro Virtual Cervantes. Acerca de la competencia comunicativa. Thesaurus Tomo XLII.Núm. 2 Recuperado de http://cvc.cervantes.es/lengua/thesaurus/pdf/42/TH_42_002_048_0.pdf.

Pogolotti, G. (15 de junio de 2019). Educación y sociedad, P. 2, en Periódico Juventud Rebelde, sábado 15 de junio de 2019, http://www.juventudrebelde.cu/opinion/2019

Roméu, A. (2007). El enfoque cognitivo, comunicativo y sociocultural de la enseñanza de la lengua y la literatura. Editorial Pueblo y Educación, La Habana.

Saborido, J. (2018). Universidad, investigación, innovación y formación doctoral para el desarrollo en Cuba Revista Cubana de Educación Superior. Versión On-line ISSN 0257-4314, vol.37 no.1. La Habana ene.abr. 2018.

Soto, J. (2016). Desde una antología del lenguaje hacia una ética intercultural de la alteridad. Amauta, Universidad del Atlántico.

Torres, P. (2016). Acerca de los enfoques cuantitativos y cualitativos en la investigación educativa cubana actual. pp. 1-15. Atenas 2 (34), Recuperado de http://atenas.reduniv.edu.cu

Trejo, M. et. al. (2015). Nuevas perspectivas en la educación superior. Trejo, María Luisa, (coordinadora). Tuxtla Gutiérrez, México: Editorial Historia Herencia Mexicana.

Trejo, M., et. al. (2019). La Maestría en Didáctica de Lenguas y el estado de arte del campo disciplinario. CD de Memorias del VII Taller La enseñanza de las disciplinas humanísticas, 2019. Universidad de Matanzas. Disponible en http://citc.umcc.cu

Unesco (2016). Texto 1: Innovación Educativa. Serie "Herramientas de apoyo para el trabajo docente" disponible en: www.unesco.org/lima 